\title{
Noise Propagation in Multipinhole SPECT Calibration
}

\author{
Kathleen Vunckx, Lin Zhou and Johan Nuyts
}

\begin{abstract}
It has been shown previously that multipinhole single photon emission computed tomography (SPECT) calibration only requires a two-point source measurement, without knowledge of the distance between them, to uniquely determine the camera geometry. In this work, we studied how this method propagates measurement noise into errors on the estimated calibration parameters, which in turn will degrade the reconstruction image quality. Furthermore, the effect on the calibration and reconstruction accuracy of fixing either the interpoint or the inter-pinhole distance during calibration is assessed. As expected, including information about the distance between the point sources improved the robustness of the calibration against measurement noise. In addition, it was found that similar robustness and even a superior spatial resolution can be obtained by fixing the distance(s) between the pinhole apertures instead of fixing those between the point sources. Finally, optimal twopoint source configurations were determined for calibrating our newly-designed 7-pinhole SPECT system for limited field of view mouse imaging while fixing the inter-pinhole distances.
\end{abstract}

\section{INTRODUCTION}

For small animal SPECT imaging using a clinical gamma camera, a calibration scan is required to determine the geometrical camera parameters with sufficient accuracy such that high spatial resolution can be achieved in the reconstruction image. A single pinhole SPECT system, performing circular camera motion, can be uniquely characterized by seven parameters [1]. It was proven mathematically and experimentally that a measurement of 3 point sources, of which at least two inter-point distances are known, is necessary and sufficient to determine these parameters. As shown mathematically in [2], [3], multipinhole SPECT only requires 2 point sources without any knowledge about their distance with respect to each other. However, anecdotal tests with measured data indicated that if the distance between the point sources is not fixed, the calibration of the multipinhole system is very senstive to noise. In this work, we investigated the influence of fixing either the point source distance(s) or the pinhole aperture distance(s) on the noise propagation.

\section{METHODS}

\section{A. Calibration Methods}

A multipinhole SPECT system with circular motion can be uniquely described by the following parameters [3]: detector distance $d d$, focal distances $f_{1, \ldots, I}$, mechanical offsets $m_{1, \ldots, I}$

The authors are with the Dept. of Nuclear Medicine, K.U.Leuven, B-3000 Leuven, Belgium.

This work is supported by F.W.O. grant G.0569.08, by IUAP grant - NIMI, by EC - FP6-project DiMI (LSHB-CT-2005-512146) and by the MoSAIC project of K.U.Leuven.

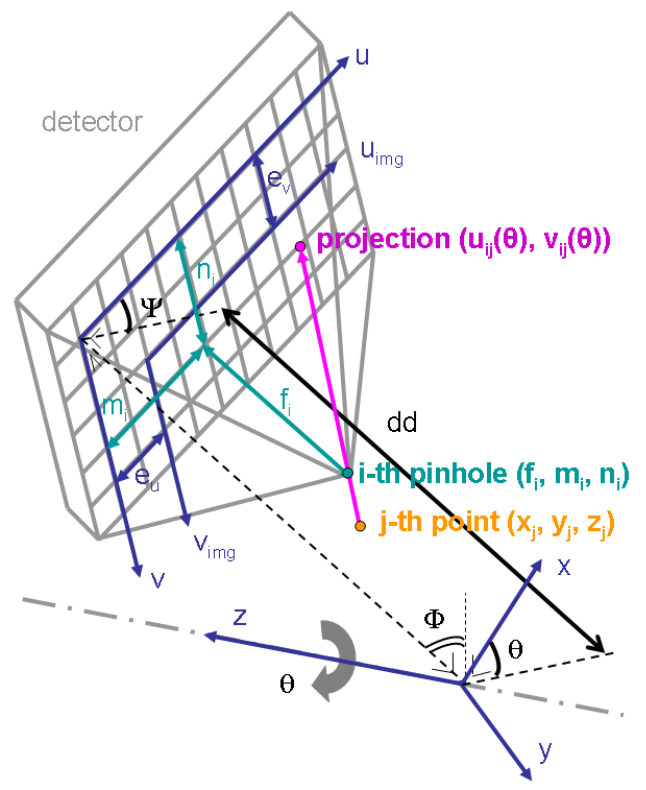

Fig. 1. Illustration of the multipinhole SPECT calibration parameters.

and $n_{1, \ldots, I}$, electrical offsets $e_{u}$ and $e_{v}$, tilt angle $\Phi$, and twist angle $\Psi$, with $I$ the total number of pinhole apertures. These parameters are briefly described in Table I and illustrated in Fig. 1 .

The projection $\left(u_{i j}(\theta), v_{i j}(\theta)\right)$ of point source $j\left(x_{j}, y_{j}, z_{j}\right)$ through aperture $i$ along projection angle $\theta$ can be written as:

$$
\begin{aligned}
u_{i j}(\theta) & =f_{i} \frac{m_{u, i}-x_{j}^{\prime \prime \prime}}{d d-f_{i}+y_{j}^{\prime \prime \prime}}+m_{u, i}+e_{u} \\
v_{i j}(\theta) & =f_{i} \frac{m_{v, i}-z_{j}^{\prime \prime \prime}}{d d-f_{i}+y_{j}^{\prime \prime \prime}}+m_{v, i}+e_{v}
\end{aligned}
$$

in which

$$
\begin{aligned}
m_{u, i} & =m_{i} \cos \Psi-n_{i} \sin \Psi \\
m_{v, i} & =m_{i} \sin \Psi+n_{i} \cos \Psi \\
{\left[\begin{array}{c}
x_{j}^{\prime \prime \prime} \\
y_{j}^{\prime \prime \prime} \\
z_{j}^{\prime \prime \prime}
\end{array}\right] } & =R_{3} R_{2} R_{1}\left[\begin{array}{l}
x_{j} \\
y_{j} \\
z_{j}
\end{array}\right]
\end{aligned}
$$




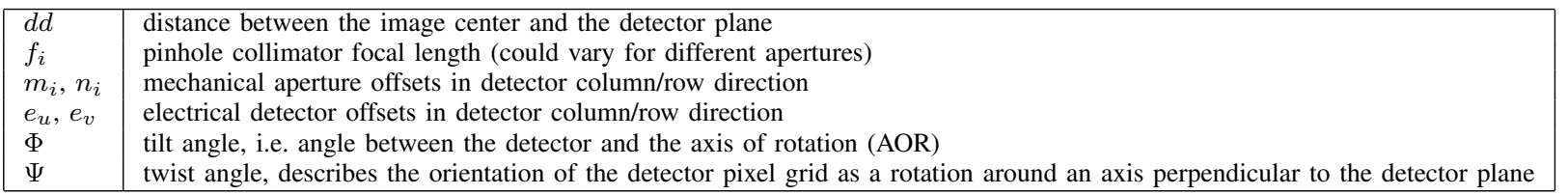

with

$$
\begin{aligned}
& R_{1}=\left[\begin{array}{ccc}
\cos \theta & \sin \theta & 0 \\
-\sin \theta & \cos \theta & 0 \\
0 & 0 & 1
\end{array}\right] \\
& R_{2}=\left[\begin{array}{ccc}
1 & 0 & 0 \\
0 & \cos \Phi & -\sin \Phi \\
0 & \sin \Phi & \cos \Phi
\end{array}\right] \\
& R_{3}=\left[\begin{array}{ccc}
\cos \Psi & 0 & -\sin \Psi \\
0 & 1 & 0 \\
\sin \Psi & 0 & \cos \Psi
\end{array}\right]
\end{aligned}
$$

For single pinhole SPECT, the distances between the three point sources could easily be fixed by expressing the coordinates of these points as a $3 \mathrm{D}$ translation, 3 rotations and 3 distances (see [4]). Similarly, two point sources or two pinhole apertures can be described as a 3D translation, 2 rotations and 1 distance, whereas if the number of sources or apertures $\mathrm{N}$ is larger than 2, a 3D translation, 3 rotations and $3 \times(\mathrm{N}-2)$ distances are required. This assumes some prior knowledge about their location. We assume the first point is located in the $x y z$ origin, the second one on the positive $x$-axis, the third one in the $x y$ half plane of non-negative $y$ coordinates. All subsequent points are assumed to have only non-negative coordinates.

\section{B. Calibration Accuracy}

Although it has been proven that a two-point source calibration can fully determine the multipinhole acquisition parameters, the parameter estimation procedure might get stuck in a local minimum due to the noise on the measurement. In this work, we study the effect of measurement noise on the accuracy of the calibration parameters and the reconstruction accuracy in a similar way as previously presented in [4]. We assume that the influence of small variations $\Delta P$ in the unknown calibration parameters $P$ on the original projection coordinates $U_{0}=\left(u_{i j}(\theta), v_{i j}(\theta)\right)$ can be approximated by the following linear system:

$$
U=U_{0}+M \Delta P
$$

where $M$ is a matrix containing the first-order derivatives of the projection coordinates $U_{0}$ (see (1) and (2)) to each parameter of $P$. The list of unknown calibration parameters depends on the calibration method: no distances fixed, interpoint distance(s) fixed, inter-pinhole distance(s) fixed, or all distances fixed. An overview is provided in Table II. When the distances between points (sources or holes) are fixed, the point configuration becomes a rigid object, which is described with the distances, translations and rotations, rather than with Cartesian coordinates.

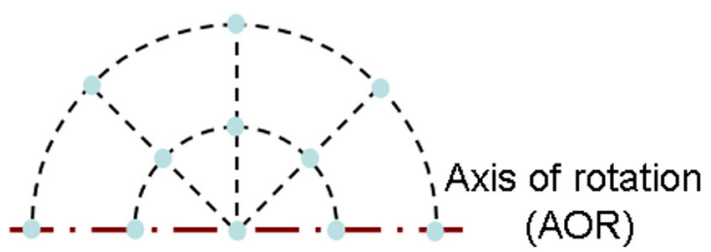

Fig. 2. Grid of point sources used to evaluate the reconstruction accuracy. The point sources are positioned on two semi-circles with a radius of $5 \mathrm{~mm} / 10 \mathrm{~mm}$ (for the 2-pinhole SPECT calibration - Sections III-A and III-B) or $10 \mathrm{~mm} / 20 \mathrm{~mm}$ (for the 7-pinhole SPECT calibration - Section III-C).

The least squares solution of the linear system of (9) yields the variations $\Delta P$ induced by small errors in the projection coordinates $\Delta U=U-U_{0}$ :

$$
\Delta P=\left(M^{T} M\right)^{-1} M^{T} \Delta U
$$

The noise on the projection coordinates is better characterized by its covariance matrix $\operatorname{cov}(U)$ than by a single noise realization $\Delta U$. The calibration accuracy can then be expressed by the covariance matrix of the estimated parameters $P$ :

$$
\operatorname{cov}(P)=\left(M^{T} M\right)^{-1} M^{T} \operatorname{cov}(U) M\left(M^{T} M\right)^{-1}
$$

\section{Reconstruction Accuracy}

As in [4], the reconstruction accuracy is evaluated by quantifying the loss of spatial resolution and the deformation in the reconstruction image. For this purpose, the grid of point sources shown in Fig. 2 is reconstructed analytically. Due to the rotational symmetry of the pinhole SPECT camera, investigating the quality of a half-plane including the axis of rotation (AOR) is sufficient.

Equations (1) and (2) can be written as a function of $X_{j}=\left[x_{j}, y_{j}, z_{j}\right]^{T}$, namely as $A X_{j}+B=0$, where $X_{j}$ is the position of the $j$-th point source of the grid. For incorrect camera parameters, this set of linear equations is typically overdetermined and hence the coordinates of the 'reconstructed' point source $X_{j}^{R}$ correspond to the least squares solution of the linear system:

$$
X_{j}^{R}=-\left(A^{T} A\right)^{-1} A^{T} B
$$

We define the loss of spatial resolution as the largest distance between the reconstructed point source and its backprojection rays. The image deformation will be specified as the largest of all displacements between the reconstructed point sources and their original positions, after a correction for a global 3D translation and rotation. These two reconstruction accuracy measures are illustrated in Fig. 3. 
TABLE II

UNKNOWN CALIBRATION PARAMETERS PER CALIBRATION METHOD.

\begin{tabular}{|l|l|l|}
\hline Calibration method & Camera-specific parameters & Point source location parameters \\
\hline No distances fixed & $d d, f_{i=1, \ldots, I}, m_{i=1, \ldots, I}, n_{i=1, \ldots, I}, e_{u}, e_{v}, \Phi, \Psi$ & $x_{j=1, \ldots, J}, y_{j=1, \ldots, J, z_{j=1, \ldots, J}}$ \\
Inter-point distance(s) fixed & $d d, f_{i=1, \ldots, I}, m_{i=1, \ldots, I}, n_{i=1, \ldots, I}, e_{u}, e_{v}, \Phi, \Psi$ & 3 translations, 2 or 3 rotations \\
Inter-pinhole distance(s) fixed & $d d, 3$ translations, 2 or 3 rotations, $e_{u}, e_{v}, \Phi, \Psi$ & $x_{j=1, \ldots, J}, y_{j=1, \ldots, J}, z_{j=1, \ldots, J}$ \\
All distances fixed & $d d, 3$ translations, 2 or 3 rotations, $e_{u}, e_{v}, \Phi, \Psi$ & 3 translations, 2 or 3 rotations \\
\hline
\end{tabular}

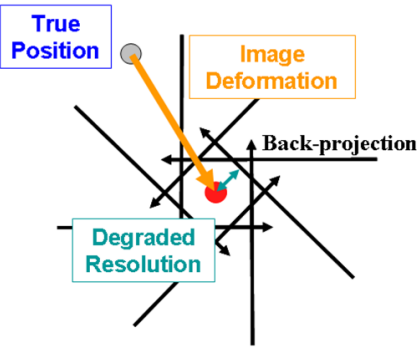

Fig. 3. Illustration of the reconstruction accuracy figures of merit: loss of spatial resolution and image deformation.

As explained in Appendix I of [4], the covariance matrix $\operatorname{cov}(P)$ can be decomposed as $\Gamma \Gamma^{T}$ to yield independent parameter estimate errors $\Delta P_{1}, \Delta P_{2}, \ldots, \Delta P_{K}$, with $K$ the total number of unknown camera-specific parameters. The point source location parameters do not influence the reconstruction, and hence can be ignored here. Because we assume that the errors have an independent effect on the image reconstruction accuracy, first all distances required to determine the loss of spatial resolution and the image deformation are calculated for each error separately. These distances are then quadratically added together, before selecting the overall largest distance:

$$
\text { accuracy }=\max \left(\sqrt{\sum_{k} \operatorname{distance}_{k}^{2}}\right)
$$

\section{Truncation Modeling}

Equations (1) and (2) yield projection coordinates for each point source through each aperture for all projection angles. However, in reality a point source will not always be detected through every aperture at every projection angle. We modeled this data truncation by only preserving the columns or rows in $U, M$ and $\operatorname{cov}(U)$ corresponding to those projection points that are located in the field of view (FOV) of the aperture and on the valid detector area (see Fig. 4).

\section{E. Optimization Method}

To optimize a two-point source calibration setup for a multipinhole SPECT system, we suggest the following four objective functions, two measuring the calibration accuracy

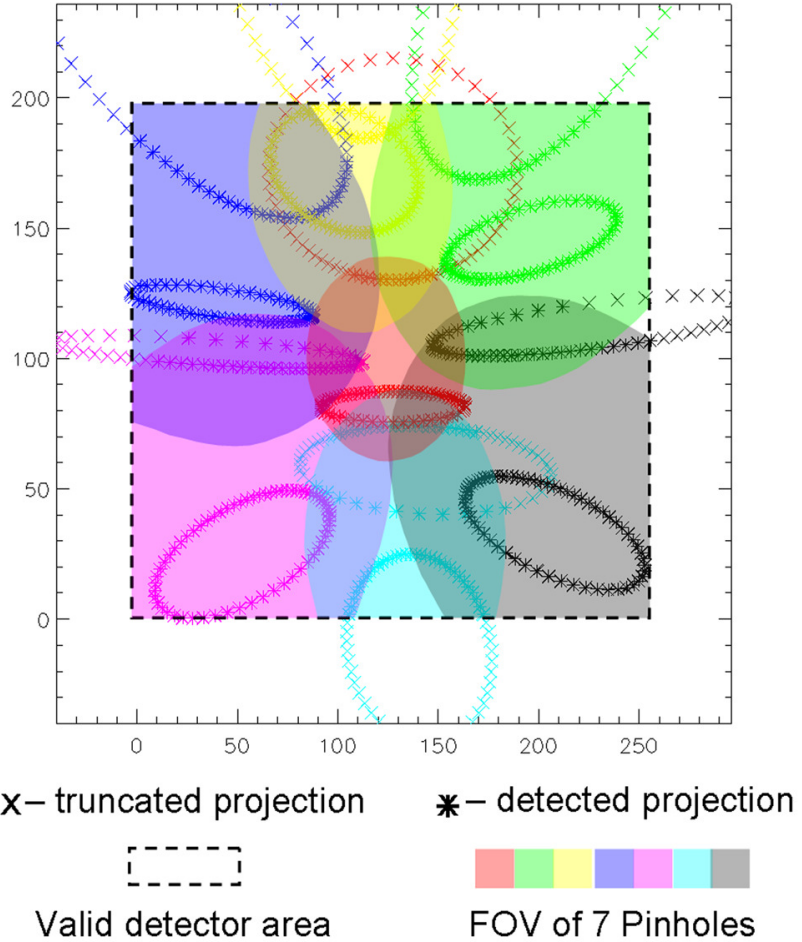

Fig. 4. Trajectory of the point source projections on the detector plane of the 2-point source configuration of Fig. 8 indicated with a star. The FOVs of the 7 apertures are shown in different colors. The valid detector area is restricted by a dashed box. Crosses indicate truncated projections, stars highlight detected projections.

and two estimating the reconstruction accuracy:

$$
\begin{aligned}
\text { Obj1 } & =\sqrt{\sum_{k=1}^{K} p_{k}^{2}} \\
\text { Obj2 } & =\sqrt{\sum_{k=1}^{K}\left(p_{k}-p_{k}^{\text {min }}\right)^{2}} \\
\text { Obj3 } & =\text { resolution loss } \\
\text { Obj4 } & =\text { image deformation }
\end{aligned}
$$

with $p_{k}$ the standard deviation of the $k$-th unknown geometryspecific parameter. $p_{k}^{\text {min }}$ is the minimum of all $p_{k}$ obtained with different calibration setups. $K$ is the total number of unknown geometrical parameters. The resolution loss and image deformation are defined as explained in Section II-C. 


\section{EXPERIMENTS}

\section{A. Noise Propagation}

To study how measurement noise, i.e. errors on the projection point coordinates, degrades the calibration and reconstruction accuracy, we investigated the calibration of a 2-pinhole SPECT system using three and two point sources and four different calibration methods (differing in which distances are fixed). The two pinhole apertures have a mechanical offset of $-10 \mathrm{~mm}$ and $10 \mathrm{~mm}$ in the column direction. The aperture plane was chosen parallel to the detector plane and to the AOR, at a distance of $25 \mathrm{~mm}$ from the image center. The pinhole collimator has a focal distance of $176 \mathrm{~mm}$. The electrical shifts, tilt angle and twist angle were set to zero. An acquisition of 64 projection images equally spaced over $360^{\circ}$ was simulated.

The three point sources of the calibration phantom were positioned at $13 \mathrm{~mm}$ from the image center each, in a direction of $-45^{\circ}, 90^{\circ}$ and $225^{\circ}$ with respect to the AOR. For the calibration phantom with only two point sources, the last point was omitted.

The coordinates of the point source projections are determined with equations (1) and (2). The measurement noise was assumed to be Gaussian distributed with a standard deviation of $0.25 \mathrm{~mm}$.

Four different calibration methods were compared: (1) fitting all parameters, including all distances, (2) fixing only the inter-point distances, (3) fixing only the inter-pinhole distance, and (4) fixing both the inter-point and inter-pinhole distances. The calibration accuracy was determined from equation (11). The diagonal elements of $\operatorname{cov}(P)$ correspond to the variances of the camera-specific parameters. In addition, the resolution loss and the image deformation were calculated as explained in Section II-C. The reconstruction accuracy was evaluated based on the grid depicted in Fig. 2, in which the two semi-circles had a radius of $5 \mathrm{~mm}$ and $10 \mathrm{~mm}$, respectively.

\section{B. Validation Study}

In this section, we verified the accuracy of the analytical method described in Sections II-B and II-C. To this end, the results of the 2-pinhole SPECT system calibration study of Section III-A were validated with repeated simulations. 250 noisy projection data sets were obtained by adding Gaussian distributed noise (mean of 0 and standard deviation of $0.25 \mathrm{~mm}$ ) to the calculated projection point coordinates, both for the three and two point source phantom. Each of these simulated measurements was used to calibrate the 2-pinhole SPECT system with the four different calibration methods.

For every method, the standard deviations on the parameter estimates were calculated and compared with the corresponding results from the analytical method as follows:

$$
\Delta_{k}(\%)=\frac{\left|p_{k}-p_{k}^{s i m}\right|}{p_{k}^{\text {sim }}} \times 100
$$

where $p_{k}$ and $p_{k}^{\text {sim }}$ represent the standard deviation of the $k$ th parameter obtained from the analytical method and from the simulations, respectively. Furthermore, the linear system approximation required zero-bias assumption. The validity

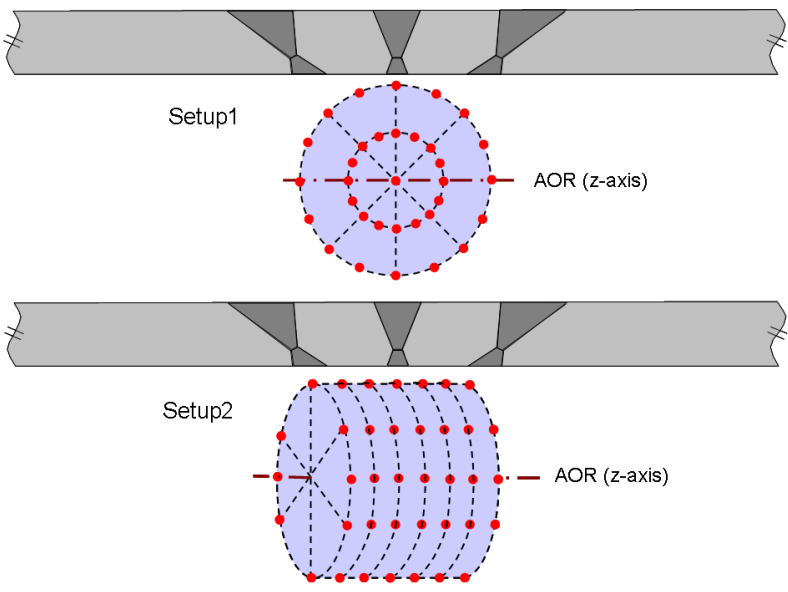

Fig. 5. Two different setups with all tested locations for a two-point source calibration. Setup 1: sampling the whole FOV, setup 2: sampling the outer surface of the animal bed.

of this assumption was also verified using these repeated simulations.

\section{Optimal 2-Point Source Calibration Setup}

The analytical methods, described in Sections II-B and II-C and validated in Section III-B, could be used to investigate the calibration and reconstruction accuracy of various 2-point source configurations used to calibrate a specific multipinhole SPECT system. In this work, we tried to determine an optimal 2-point source configuration to calibrate our newly-designed 7pinhole SPECT system [5], using fixed inter-pinhole distances during calibration. The collimator consists of a central aperture with 6 surrounding apertures located on a circle with a radius of $20 \mathrm{~mm}$. All pinholes have a diameter of $1.5 \mathrm{~mm}$ and focus at a distance of $27.5 \mathrm{~mm}$ or $35 \mathrm{~mm}$. A more detailed description, as well as a technical drawing can be found in [5]. We optimized the calibration setup for our typical acquisition settings of 64 projections equally spread over $360^{\circ}$. To model truncation, we assumed that the projections were acquired in a matrix of $256 \times 200$ with $1.95 \mathrm{~mm}$ square pixels. The electrical shifts, tilt and twist angle were set to zero. The error in the determination of the center of the projection points due to measurement noise was modeled by a Gaussian distribution with a standard deviation of $0.25 \mathrm{~mm}$.

We propose two calibration setup approaches. In the first one, we assumed that the point sources can be positioned anywhere (as in a separate calibration scan). Hence, we sampled the spherical FOV by 4 times 2 concentric circles with a radius of 10 and $20 \mathrm{~mm}$ rotated over $0,45,90$ and $135^{\circ}$ around the AOR (see Fig. 5 (Setup 1)). Each circle consists of 16 points. The image center was also considered as a candidate. The calibration and reconstruction accuracy induced by any combination of two of these point sources were evaluated and the best configurations were determined using the four proposed objective functions (equations (14)(17)). The grid used to evaluate the reconstruction accuracy consisted of two semi-circles with radii of $10 \mathrm{~mm}$ and $20 \mathrm{~mm}$, respectively (see Fig. 2). 


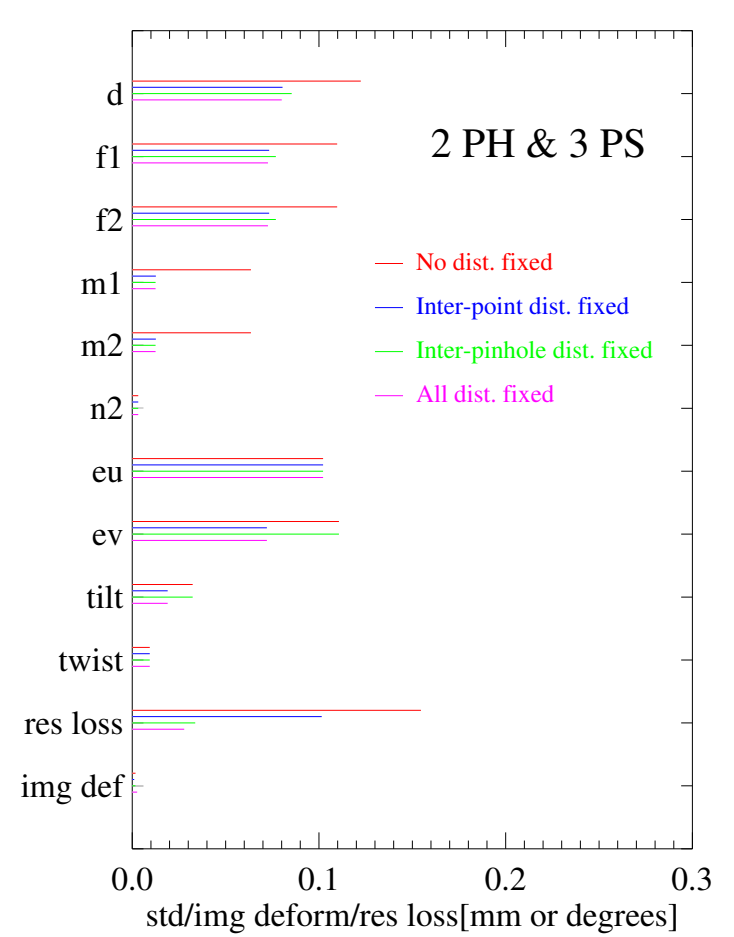

Fig. 6. Calibration and reconstruction accuracy of the 2-pinhole SPECT system described in Section III-A using four different 3-point source calibration methods. Standard deviations on the camera-specific parameters are expressed in $\mathrm{mm}$ or degrees; resolution loss and image deformation are expressed in $\mathrm{mm}$.

In the second approach, we only allowed point sources for calibration purposes to be attached to the animal bed, which is a cylindrical tube. Therefore, we sampled the surface of a cylinder with a radius of $20 \mathrm{~mm}$, length of $30 \mathrm{~mm}$ and central axis equal to the AOR. 56 possible point source locations are equally distributed over 7 circles, which are perpendicular to the AOR and $-15,-10,-5,0,5,10$ and $15 \mathrm{~mm}$ off-center (see Fig. 5 (Setup 2)). Again, all combinations of 2 point source locations were evaluated.

\section{RESUlts}

\section{A. Noise Propagation}

Fig. 6 and 7 show the results of the noise propagation experiments, described in Section III-A. If we compare 3point source to 2-point source calibration (Fig. 6 vs. Fig. 7), we can conclude that both the calibration and reconstruction accuracy are improved if a point source is added and the same calibration method is used. Especially the electrical shifts and the tilt angle were more accurately estimated. For both the 3- and 2-point source calibration, including information about either the inter-point (blue lines) or inter-pinhole distances during calibration (green lines), or both (pink lines), reduced the standard deviations of the detector distance and the focal distances, but more substancially those of the mechanical distances in the column direction, compared to fitting all distances (red lines). In addition, $e_{v}$ and the tilt angle were also estimated with a higher accuracy when using the 3-point

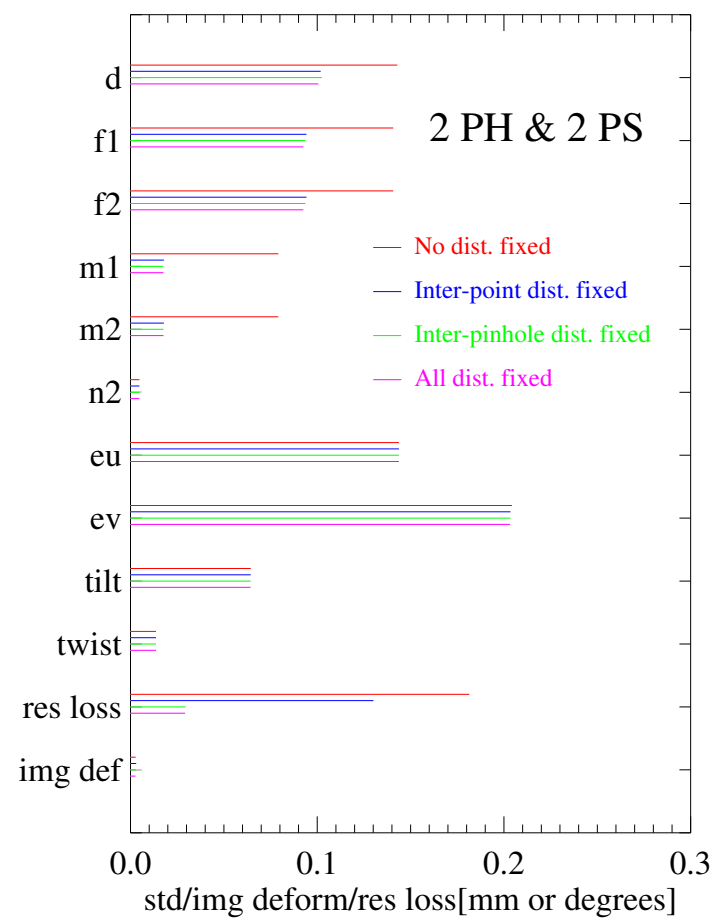

Fig. 7. Calibration and reconstruction accuracy of the 2-pinhole SPECT system described in Section III-A using four different 2-point source calibration methods. Standard deviations on the camera-specific parameters are expressed in $\mathrm{mm}$ or degrees; resolution loss and image deformation are expressed in $\mathrm{mm}$.

source calibration with inter-point distances fixed (blue and pink lines).

The resolution loss decreased when keeping the inter-point distance(s) fixed. However, fixing the inter-pinhole distance appeared to be far more effective to improve the spatial resolution. The image deformation was very small in all cases.

\section{B. Validation Study}

First, the standard deviation on each camera-specific parameter estimated from the analytical method is compared with the one derived from the simulations. In most cases, $\Delta_{k}<6 \%$. The only exception was for the twist angle when using 2 point sources, where $\Delta_{k} \approx 10 \%$.

Second, the linear system that we used to evaluate the calibration accuracy (Section II-B, Eq. (9)) implicitly assumes that the parameter estimates have zero bias. Actually, the bias calculated from the simulations are indeed always close to 0 $(<0.015)$, no matter which point source configuration (2-point or 3-point) was used or which method (fixing/fitting either of the distances) we applied in the calibration. Combining this with the estimated standard deviations, it indicates that the mean values are always very close to the correct values, comfirming the zero-bias assumption of the linear system.

\section{Optimal 2-Point Source Calibration Setup}

1) Without Truncation: In Fig. 8(a), the three best point source combinations for setup 1 are highlighted by a solid line. They all nearly minimize all four objective functions 


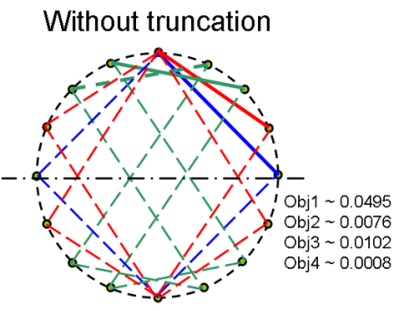

(a)

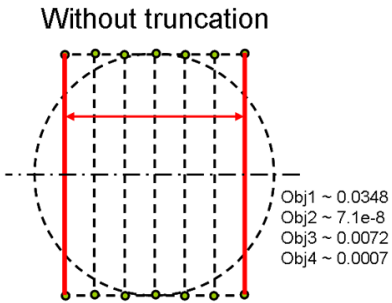

(c)

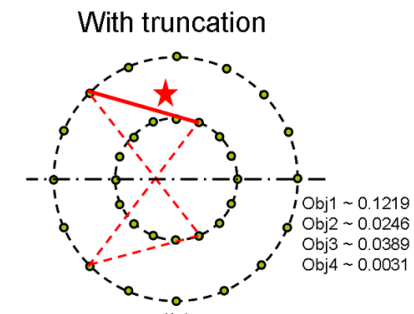

(b)

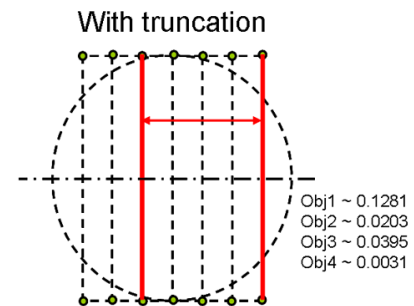

(d)
Fig. 8. Overview of the 2-point source configurations yielding the best calibration and reconstruction accuracy (based on the 4 objective functions presented in Section II-E). Top row: results for setup 1 (Fig. 5(top)), bottom row: results for setup 2 (Fig. 5(bottom)). Results are shown for calibration without (left column) and with truncation modeling (right column). Solid lines with different colors indicate point source configurations with almost the same accuracy. Point sources linked with dashed lines are equivalent to combinations indicated with a solid line of the same color.

(14)-(17) if truncation is not taken into account. The different colors indicate different point source combinations which yield almost the same accuracy. The dashed lines link calibration setups that are equivalent to those connected by a solid line of the same color. As can be seen, the results are highly symmetrical with respect to the central plane, i.e. the plane perpendicular to the AOR and containing the image center. In addition, because we modeled a perfect circular camera motion of $360^{\circ}$, each selected point source location can be replaced by any other location on the circle through the initial location, with its origin on the AOR and positioned in a plane perpendicular to the AOR, without losing any accuracy. The best 2-point source combinations have a rather large axial spacing and at least one point has to be located far from the AOR.

If both point sources have to be attached to the animal bed, as in setup 2, they are preferred to be located at the maximum distance measured along the AOR (see Fig. 8(c)). As was the case for the optimal combinations of setup 1, any point of the left most outer circle can be combined with any point of the right most outer circle to yield the same optimal accuracy.

2) With Truncation: In reality, however, a point source can be located outside the FOV of a pinhole or can be projected outside the active detector area. This results in truncated projection data, as shown in Fig. 4. Hence, the optimal 2point source combinations found above, are not necessarily the optimal combinations for a real measurement. Indeed, from Fig. 8(b) we can see that a different optimal calibration setup was found when truncation was modeled. Because the multipinhole design, and therefore also the truncation, is asymmetrical, two setups symmetrical with respect to the central plane are no longer equivalent. The general criteria that the axial distance should be sufficiently large and that at least one point should be far from the AOR remain valid, but point sources are now restricted to locations that are visible from sufficient projection angles.

For the second setup, the same conclusions can be drawn. The axial distance between the point sources had to be reduced to guarantee that sufficient projection points are available to ensure accurate calibration.

\section{Discussion}

From the results of the noise propagation study, presented in Section IV-A, we recommend to fix the inter-point or inter-pinhole distances during calibration, whenever possible. In addition, using more than two point sources provides extra robustness. However, increasing the number of point sources might lead to difficulty in differentiating the different point source projections, especially if the number of pinhole apertures is high. Simultaneous projection point identification and parameter fitting can alleviate this problem, as explained in [6].

Regardless of the calibration method used, the maximum standard deviations on the calibration parameter estimates were about $0.2 \mathrm{~mm}$ and $0.07^{\circ}$. The addition of a point source and the use of distance information seem to have complementary effects on the calibration accuracy. The former mainly improves the electrical shifts and the tilt angle, whereas the latter mainly influences the mechanical shifts, and the detector and focal distances. The resolution loss benefits from both, although fixing the inter-pinhole distances has the most dominant effect. The image deformation improves most from an extra point source, but its error was already negligible.

The accuracy of the estimate of $n_{1}$ was not shown, because it is not fitted during calibration, but calculated from $n_{1}=m_{1} \tan \Psi$. This was done to determine the location of the origin of the coordinate system along the AOR.

For a simultaneous animal-calibration scan, it is often more straightforward to know the distances between the apertures accurately, than the distances between the point sources. Indeed, typically multiple apertures are drilled or eroded with high precision in a single rigid plate [7] or cylinder [8]. Hence, once the distances are known, they will be known for every scan with this collimator. Point sources can either be combined in a rigid phantom, or attached, e.g., to the animal bed as separate markers. In the former case, the interpoint distances should stay constant as long as the phantom is sufficiently rigid. Usually, this restricts the shape of the phantom, possibly compromising its use during the animal scan. This was e.g. the case for our calibration phantom, hence demanding a separate calibration scan. When using individual markers, special tricks are required to guarantee an accurately known fixed distance between them, e.g. attaching point sources in grooves machined on the surface of a plastic cylinder [9].

To determine the inter-pinhole distances accurately, one could either trust the specifications provided by the manufacturer, or acquire a large number of calibration scans, e.g., using a calibration phantom with point sources at accurately known 
distances, and derive the distances between the apertures by averaging over all calibrations (performed with fixed interpoint distances).

Perfoming a simultaneous animal and calibration scan has two main advantages. Firstly, because the calibration data are acquired at the same time as the animal data, errors induced by variation in camera motion between two subsequent scans using the same protocol are avoided by definition. Secondly, as it eliminates the need to acquire an additional scan before or after each (series of) animal scan(s), it could often reduce the total scan time significantly.

To facilitate discrimination between point source and animal projections, the use of two isotopes with sufficiently different energies is recommended. DiFilippo et al. suggested the use of Gadolinium-153 $\left({ }^{153} \mathrm{Gd}\right)$, which emits among others gammas of $97 \mathrm{keV}$ and $103 \mathrm{keV}$ (no higher-energy emissions) [9]. These energies can easily be discriminated from the photopeak energies of the most commonly used SPECT isotopes $\left({ }^{99 \mathrm{~m}} \mathrm{Tc}\right.$ : $140 \mathrm{keV},{ }^{123} \mathrm{I}$ : $159 \mathrm{keV}$ ). Additional advantages of using two distinct isotopes are the lower dose required to discern the point sources and the fact that a long-living isotope can be chosen, e.g., ${ }^{153} \mathrm{Gd}$ with a half-life of 242 days or 8 months, such that the point sources do not need to be refilled for every scan.

For modeling the truncation, we only used a mask to delineate the FOV of each pinhole aperture. The sensitivity for each point source location was ignored, because we assumed that a point source will be chosen that is sufficiently active to be detected.

Truncation due to the limited detector area and pinhole FOV strongly influences the noise propagation. Hence, different optimal 2-point source configurations were found when truncation was modeled or not. Without truncation modeling, the two points were positioned as far apart as possible, with at least one point located very far from the AOR. If truncation is taken into account, a compromise is required between these conditions and a sufficiently high number of valid point source projections. For the realistic case where truncation is modeled, only small differences are observed in the optimized values of the four objective functions for the two setups. The resolution loss is limited to $0.04 \mathrm{~mm}$ and the image deformation was only $0.0031 \mathrm{~mm}$, which are both very small with respect to the systems spatial resolution of about $1.2 \mathrm{~mm}$. Hence, suitable configurations for attaching 2 point sources at the animal bed were found.

So far, we only processed measured data using subsequent calibration data. From the same calibration scan, we performed both 3- and 2-point source calibrations, with either fixed inter-point or inter-pinhole distances, or without fixing any distance. Preliminary results provided visually very similar reconstruction images when using data of 3 or 2 point sources, as well as when using information about inter-point or interpinhole distances. Calibration without any distance information appeared to be less stable, as in some cases the phantom dimensions were scaled, probably because of large errors on the estimated focal distances, detector distance and mechanical offsets. More thorough analysis of phantom data is required.

\section{CONCLUSION}

The noise propagation in multipinhole SPECT was studied by comparing the application of various 3- and 2-point source calibration methods. Similar calibration and reconstruction accuracy could be obtained by including information about either the inter-point source or the inter-pinhole distances during calibration. Not including any distance information or reducing the number of point sources decreased the robustness. Various 2-point source configurations were evaluated for calibrating our newly-designed 7-pinhole SPECT system for limited FOV mouse imaging and the best setups were found to consist of 2 point sources, positioned such as to ensure a maximum axial distance between each other, but also minimum projection truncation. At least one point should be located far from the AOR. These conditions facilitate a simultaneous animal and calibration scan, where two or more point sources are attached to the animal bed. This could often substantially reduce the total acquisition time and circumvents possible inaccuracy due to inter-scan variability.

\section{REFERENCES}

[1] D. Bequé, J. Nuyts, G. Bormans, P. Suetens, and P. Dupont, "Characterization of pinhole SPECT acquisition geometry." IEEE Trans. Med. Imag., vol. 22(5), pp. 599-612, 2003.

[2] Y. C. Wang and B. M. W. Tsui, "Pinhole SPECT with different data acquisition geometries: Usefulness of unified projection operators in homogeneous coordinates." IEEE Trans. Med. Imag., vol. 26(3), pp. 298 $308,2007$.

[3] K. Vunckx, M. Defrise, D. Bequé, C. Vanhove, A. Andreyev, and J. Nuyts, "Geometrical calibration and aperture configuration design in multi-pinhole SPECT." IEEE Internat. Symp. on Biomed. Imag., pp. 1403-1406, 2008.

[4] D. Bequé, J. Nuyts, P. Suetens, and G. Bormans, "Optimization of geometrical calibration in pinhole SPECT." IEEE Trans. Med. Imag., vol. 24(2), pp. 180-190, 2005.

[5] K. Vunckx, J. Nuyts, B. Vanbilloen, M. De Saint-Hubert, D. Vanderghinste, D. Rattat, F. M. Mottaghy, and M. Defrise, "Optimized multipinhole design for mouse imaging." IEEE Trans. Nucl. Sci., vol. 56(5), pp. 2696$2705,2009$.

[6] J. Nuyts, K. Vunckx, M. Defrise and C. Vanhove, "Small animal imaging with multi-pinhole SPECT." Methods, vol. 48(2), pp. 83-91, 2009.

[7] N. U. Schramm, G. Ebel, U. Engeland, T. Schurrat, M. Behe, and T. Behr, "High-resolution SPECT using multipinhole collimation." IEEE Trans. Nucl. Sci., vol. 50(3), pp. 315-320, 2003.

[8] F. J. Beekman and B. Vastenhouw, "Design and simulation of a highresolution stationary SPECT system for small animals." Phys. Med. Biol., vol. 49(19), pp. 4579-4592, 2004.

[9] F. P. DiFilippo, M. J. Riffe, K. M. Harsch, N. P. McCabe and W. D. Heston, "Detached multipinhole small animal SPECT device with real-time calibration." IEEE Trans. Nucl. Sci., vol. 53(5), pp. 2605-2612, 2006. 